\section{Transfer from free-recall pair learning to free-recall sentence learning}

\author{
JAMES H. CROUSE, University of Dela- \\ ware, Newark, Del. 19711
}

Transfer from free-recall pair learning to free-recall sentence learning was studied in a 3 by 3 factorial experiment. The first variable was the intrapair sequence of the List 1 pairs; noun-verb, verb-noun, or noun-noun. The pairs in a given list entered an $A-B, A-B$, an $A-B, C-D$, or an $A-B, A-B$, transfer paradigm with the corresponding sequence in the noun-ierb-noun sentences in List 2, therefore transfer paradigm was the second variable. The major finding was that. in general, sentence leaming was fastest in the $A-B, A-B$ paradigm, intermediate in the $A-B, C-D$ paradigm, and slowest in the $A-B$, $A-B_{r}$ paradigm. These transfer results paralleled the findings of other transfer studies, and were interpreted in terms of Ss using the learned sequences from pair leaming to integrate the sentences. Analy sis of intrasentence sequence acquisition sug. gested that the mamner in which the sequences are used to integrate the sentence may be quite complex.

In the present experiment two free-recall tasks were learned in succession. The items in the first task were word pairs and in the second were simple noun-verb-noun sentences (e.g., Tigers Eat Meat). The purpose of the experiment was to determine transfer from free-recall pair learning to free-recall sentence learning.

In the first task the items consisted of pairs in which the intrapair sequence for every pair within a list formed an $A-B, A-B$, an $A-B, C-D$, or an $A-B, A-B_{r}$ transfer paradigm with the words from the same intrasentence sequence in the noun-verbnoun sentences. If transfer parallels that commonly found in these paradigms (cf. Martin, 1965), then sentence learning will be fastest when the List 1 pairs form an $A-B$, $A \cdot B$ transfer paradigm with the words from the corresponding sequences of the sentences, learning will be intermediate when the pairs form an A-B, C-D paradigm, and slowest when they form an A-B, A-B paradigm.

\section{DESIGN}

One of nine lists of paired words was learned during Stage 1, and during Stage 2 Ss were transferred to the same list of sentences. The nine first-lists represented the factorial combinations of the 3 by 3 design. The first variable was the intrapair sequence of the List 1 pairs; all noun-verb (N.V), verb-noun (V-N), or noun-noun (N-N) pairs. The pairs in a given list entered a transfer relationship with the words from the same intrasentence sequence in the List 2 sentences, e.g., the N.V pairs entered a transfer relationship with the N-V sequences in the sentences. The second variable was the transfer paradigm formed by the List 1 pairs and the corresponding List 2 sequences, either an A-B, A-B paradigm (Condition $A \cdot B)$, an $A \cdot B, \quad C \cdot D$ paradigm (Condition C-D), or an $A \cdot B, A \cdot B_{I}$ paradigm (Condition $A \cdot B_{\mathrm{r}}$ ). Sixteen Ss were randomly assigned to each of the nine conditions formed by the orthogonal combinations of intrapair sequence in List 1 and transfer paradigm.

\section{MATERIALS}

List 2 consisted of 12 sentences conforming to the noun-verb-noun structure. The mean Lorge-count frequency (Thomdike \& Lorge, 1944) was 438.50 , 540.25 , and 528.61 for the nouns, verbs, and nouns, respectively, in the list. The meaningful similarity of the words and the sentences was kept as low as inspection would allow. The first lists in Condition A-B were constructed from the sentences by pairing the two appropriate words from each sentence, i.e., noun and verb in the N.V condition, verb and noun in the V-N condition, and noun and noun in the N.N condition. In Condition $A-B_{r}$ the first lists were constructed by re-pairing these appropriate words from the sentences in such a manner that, by inspection, the two words in each pair had the same plausibility of occurring together in a sentence as in the other first lists. The first lists in Condition C.D were chosen by first constructing 12 new noun-verb-noun sentences by the same criteria as the List 2 sentences. The mean Lorge-count frequency was 464.50 , 576.75 , and 565.00 for the nouns, verbs, and nouns, respectively, and the meaningful similarity of these words and sentences to the List 2 words and sentences was kept as low as inspection would allow. From these sentences, the first lists were constructed by pairing the proper words from each sentence, i.e., noun and verb in the N.V condition, etc. Three presentation orders were used for each list.

\section{PROCEDURE}

The first-list pairs were learned to a criterion of one perfect recitation by the free-recall method. On the study portion of cich trial the pairs were presented at a t-ses rate. The test portion of each trial was also paced with the drum turned at a 4-sec rate and with asterisks signifying the successive recalls. The Ss were instructed to recall a different pair at each turn of the drum and told that it did not matter which pair they recalled for a given asterisk. They were told that for a pair to be counted as correct they had to recall both words in the correct order which was left to right as typed on the drum. Approximately 3 min after the criterion was reached, nine trials were given on the sentence list by the free-recall method. The sentence list was presented in exactly the same manner as List 1 and the Ss now were told that for a sentence to be correct the three words had to be given in correct order.

\section{RESULTS}

List 1 Learning

The mean trials to criterion for the nine groups ranged between 8.63 and 12.88 , and the $F(8,135)=1.86$ was not significant.

\section{List 2 Learning}

Since the transfer paradigms formed a relationship between a List 1 intrapair sequence and words from the same intrasentence sequence, it is appropriate to examine noun-verb (N.V), verb-noun (V-N), and noun-noun (N-N) intrasentence sequence acquisition. This is done by computing the conditional response probability (CRP) for each intrasentence sequence. The CRP for an intrasentence sequence is the probability of the second word being correct given that the first word is correct. Each S's List 2 learning was scored to give credit for any word that was correct, and for each $S$ the $C R$ frequency for the $\mathrm{N} \cdot \mathrm{V}, \mathrm{V} \cdot \mathrm{N}$, and $\mathrm{N} \cdot \mathrm{N}$ intrasentence sequence was summed over all sentences and List 2 trials. The CRP was then determined for each intrasentence sequence by dividing the $\mathrm{CR}$ frequency for the sequence by the number of times the first word in the sequence was correct. The mean CRP for each intrasentence sequence as a function of transfer paradigm and intrapair sequence is presented in Table 1, and the major findings are as follows: (1) With only two exceptions, CRPs were highest in Condition A.B

Table 1

Mean Conditional Response Probability (CRP) For Each Intrasentence Sequence

\begin{tabular}{ccccc} 
Intrapair & \multicolumn{4}{c}{ Intrasentence Sequence } \\
Sequence & $\begin{array}{c}\text { Transter } \\
\text { Paradigm }\end{array}$ & N-V & V-N & N-N \\
N-V & A-B & .990 & .995 & .993 \\
& C-D & .964 & .990 & .987 \\
& A-B & .925 & .985 & .969 \\
V-N & A-B & .992 & .996 & .994 \\
& C-D & .944 & .994 & .992 \\
& A-B & .956 & .994 & .990 \\
N-N & A-B & .980 & .999 & .998 \\
& C-D & .959 & .992 & .987 \\
& A-B & .935 & .988 & .975 \\
\hline
\end{tabular}


Table 2

Mean Number of Correct Sentences on Trial 1 and Trials $1-9$

\begin{tabular}{lrcc}
$\begin{array}{l}\text { Transfer } \\
\text { Paradigm }\end{array}$ & N-V & $\begin{array}{c}\text { Trial 1 } \\
\text { Intrapair Sequence } \\
\text { V-N }\end{array}$ & N-N \\
\hline A-B & 10.25 & 7.75 & 7.81 \\
C-D & 4.81 & 4.56 & 4.06 \\
A-B & 4.44 & 4.75 & 5.63 \\
& & Trials $1-9$ & \\
A-B & 100.93 & 92.19 & 98.56 \\
C-D & 82.38 & 82.50 & 82.38 \\
A-B & 82.00 & 81.13 & 89.75 \\
\hline
\end{tabular}

intermediate in Condition C-D, and lowest in Condition $A-B_{\mathrm{r}} \quad[\mathrm{F}(2,135)=18.25]$. Each of the individual comparisons was also significant; the $\mathrm{Fs}(1,135)$ were 12.22 , 36.21 , and 6.35 for the $A \cdot B$ vs $C \cdot D, A \cdot B$ vs $A-B_{r}$, and $C-D$ vs $A-B_{r}$ comparisons, respectively. (2) The difference among the transfer paradigms was larger for the N.V intrasentence sequence than the other sequences $[F(4,270)=13.82$ for the Transfer Paradigm by Intrasentence Sequence interaction]. (3) The effect of the transfer paradigms on acquisition of an intrasentence sequence did not depend on whether the first-list intrapair training was for that intrasentence sequence or not, as indicated by the nonsignificant Transfer Paradigm by Intrapair Sequence by Intrasentence Sequence interaction $[F(8,270)=1.95]$. (4) Acquisition was always lower for the N.V intrasentence sequence than for the other sequences $[F(2,270)=100.84]$.

Measures of overall learning are given by the mean number of correct sentences recalled by each group on Trial 1 and over Trials 1-9. Both show essentially the same findings (Table 2). (1) Transfer paradigm was a significant variable in both analyses $[\mathrm{Fs}(2,135)=72.59$ and 44.45 , respectively]. In both analy ses Condition A-B was superior to both Condition C-D [Fs( 1,135$)$ $=120.82$ and 74.96 , respectively 1 , and Condition A-B $\mathrm{B}_{\mathrm{r}}[\mathrm{Fs}(1,135)=95.46$ and 57.19 , respectively]. There was little difference, however, between Conditions C.D and A-B $[F s(1,135)=1.49$ and 1.20 , respectively] . (2) The Transfer Paradigm by Intrapair Sequence interaction was significant in both analyses $[\mathrm{Fs}(4,135)=4.72$ and 2.44, respectively], and reflected the following pattern. The difference between Conditions $\mathrm{A}-\mathrm{B}$ and $\mathrm{A}-\mathrm{B}_{\mathrm{r}}$ was substantial for each intrapair sequence, but was largest for the N.V sequence. In addition, while there was very little difference between Conditions $C-D$ and $A-B_{r}$ for the N.V and V-N intrapair sequences, Condition $A-B_{r}$ was somewhat higher for the N-N sequence.

\section{DISCUSSION}

The transfer paradigm findings parallel those found in other transfer studies (cf. Martin, 1965) in several important ways. First, prior acquisition of N-V,V-N, and N-N intrapair sequences in Condition A-B resulted in faster sentence learning by every measure than prior acquisition of the sequences in Condition $A-B_{r}$. Since transfer of item familiarization is held constant between these two conditions, the difference in transfer can be attributed to transfer of the learned sequences. Therefore, appropriate sequences learned in Condition A-B facilitate subsequent integration of the sentences more than inappropriate sequences learned in Condition $A-B_{r}$. Second, in comparison with Condition C-D, sentence learning was faster in Condition $A-B$ and slower in Condition $A-B_{r}$ for all measures except for overall sentence learning on Trial 1 and over Trials 1-9 where no decrement in Condition $\mathrm{A}-\mathrm{B}_{\mathrm{r}}$ was found. These comparisons with Condition C-D must be interpreted in view of differential transfer-of-item familiarization which would tend to inflate facilitation in
Condition A-B and mask decrements in Condition $A-B_{r}$. Therefore, the results of the comparisons with Condition C.D can be attributed to transfer of the learned sequences. However, the most impressive finding was that prior acquisition of inappropriate $\mathrm{N} \cdot \mathrm{V}, \mathrm{V}-\mathrm{N}$, and $\mathrm{N}-\mathrm{N}$ intrapair sequences in Condition $A-B_{r}$ produced lower CRPs than irrelevant sequences in Condition C-D in spite of any transfer of item familiarization that would have tended to mask this decrement.

The transfer findings in this study suggest an explanation in which $\mathrm{Ss}$ use the sequences learned in Task 1 to integrate the sentences during Task 2. The manner in which the sequences are used to integrate the sentences during Task 2 may be quite complex. Most conceptions of sequence usage would have acquisition of the N.V intrasentence sequence affected primarily by training on the $\mathrm{N}-\mathrm{V}$ intrapair sequence, and acquisition of the $\mathrm{V}-\mathrm{N}$ intrasentence sequence affected primarily by training on the $\mathrm{V} \cdot \mathrm{N}$ intrapair sequence, and the N-N intrasentence sequence affected primarily by training on the $\mathrm{N} \cdot \mathrm{N}$ intrapair sequence. However, the CRP measure showed that the N.V intrasentence sequence was always most affected by the transfer paradigms regardless of the intrapair sequence on which training was given. Therefore, the sentences in the present study appear to be integrated in such a manner that the transfer of any acquired sequence affects primarily the acquisition of the N.V intrasentence sequence.

\section{REFERENCES}

MARTIN, E. Transfer of verbal paired associates. Psychological Review, 1965, 72, 327-343.

THORNDIKE, E. L.. \& LORGE, I. The teachers word book of 30,000 words. New York: Columbia University Press, 1944. NOTE

1. Appreciation is expressed to Richard $\mathrm{K}$. Landon who assisted in the collection of the data.

\section{Notes \& News}

The American Journal of Psychology has decreased the time required for editorial decisions and now has a publication lag of only a few months. Manuscripts may be sent to Lloyd G. Humphreys, Department of Psychology, University of Illinois, or to one of the other principal editors listed in the journal.

The Tenth Annual Meeting of the Psychonomic Society will be held at the Chase-Park Plaza Hotel in St. Louis, Nov. 6, 7, 8, 1969. A Call for Papers has been mailed to members. Those who are not members may present papers when sponsored by a member. Hence, a non-member who wishes to present a paper should contact a member, secure a form, fill it out and have it signed by the member-sponsor. Deadline for Abstracts will be August 1, 1969. Abstracts as well as requests for further information on the meetings should be sent to Psychonomic Society, Inc., 1200 West 34th St., Austin. Texas, 78705. The complete program of the meetings will be published in September as a supplementary issue to Psychonomic Science. It will, of course, be distributed to members who are not subscribers to this Journal. 